\title{
Appendix A: Language activism strategy framework
}

The language activism strategy framework consists of 3 central actions targeted towards 5 goals. Actions may be influenced by any combination of 6 key characteristics. These characteristics are represented as scales, and different activism strategies can be described through their relative positions on these scales. This framework is intended to describe a range of strategic actions, and does not imply the superiority of strategies with certain targets nor certain characteristics. Each strategy is embedded in a specific language ecology which shapes and informs the who, what, and how of language activism, and enables or constrains the outcomes. Different strategies will thus be more or less possible and desirable depending on the context and the actors involved.

\begin{tabular}{ll}
\hline Actions & Goals \\
\hline- Creating & - Resources \\
- Connecting & - Events \\
- Representing & - Spaces/Structures \\
& - People/ Identities \\
& - Communication practices \\
\hline
\end{tabular}

\begin{tabular}{llll}
\hline Characteristic & Scale & & \\
\hline Location: & Local & $\leftrightarrow$ & International \\
Timeframe: & Slow & $\leftrightarrow$ & Rapid \\
Visibility \& mobility: & Low & $\leftrightarrow$ & High \\
Socio-historical orientation: & Future & $\leftrightarrow$ & Past \\
Diversity orientation: & Syncretic & $\leftrightarrow$ & Purist \\
Participation: & Open participation & $\leftrightarrow$ & Controlled participation \\
\hline
\end{tabular}


